\title{
Association between time to lithotripsy and stone-free rate in patients with ureteral stones undergoing shock wave lithotripsy
}

\author{
Satoshi Washino ${ }^{1,2} \cdot$ Takanori Hayase $^{2} \cdot$ Tomoaki Miyagawa $^{2} \cdot$ Yoshiaki Arai $^{1}$
}

Received: 26 July 2020 / Accepted: 18 November 2020 / Published online: 2 January 2021

(c) The Author(s), under exclusive licence to Springer-Verlag GmbH, DE part of Springer Nature 2021

\begin{abstract}
Early shock wave lithotripsy is associated with higher stone-free rate compared to delayed treatment of ureteral stones, but may constitute overtreatment because ureteral stones can pass spontaneously. We studied the association between time to treatment and stone-free rate in patients with ureteral stones to determine optimal shock wave lithotripsy timing. We retrospectively analyzed 537 patients undergoing shock wave lithotripsy for ureteral stones. Patients were divided into five groups according to time from onset of symptoms to lithotripsy—urgent (0-3 days), early (4-30 days), late (31-60 days), long-delayed lithotripsy ( $\geq 61$ days), and asymptomatic. Stone-free rates were compared among groups. Mean age and stone size were $55.6 \pm 13.1$ years and $7.48 \pm 3.29 \mathrm{~mm}$, respectively. Mean number of shock wave lithotripsy sessions and stone-free rate were 1.37 and $91.6 \%$, respectively, in the overall population. Stone-free rates were $95.2 \%, 96.8 \%, 91.3 \%, 86.3 \%$, and $82.7 \%$ in urgent, early, late, long-delayed lithotripsy, and asymptomatic groups, respectively. Long-delayed lithotripsy and asymptomatic groups had significantly more lithotripsy sessions and lower stone-free rate, compared to urgent and early lithotripsy groups. In multivariate analysis, time to lithotripsy [long-delayed lithotripsy (odds ratio: $0.273, p=0.004$ ) and asymptomatic nature (odds ratio: $0.236, p=0.002$ )] and age (odds ratio: $0.959, p=0.003$ ) independently affected stone-free rate. In conclusion, time to lithotripsy is a strong predictive factor for stone-free status following shock wave lithotripsy. Urgent shock wave lithotripsy did not improve stone-free rate if performed within 1 month. However, time to shock wave lithotripsy $>2$ months reduced likelihood of stone-free status.
\end{abstract}

Keywords Shock wave lithotripsy $\cdot$ Stone-free rate $\cdot$ Time to lithotripsy $\cdot$ Ureteral stones

Supplementary Information The online version contains supplementary material available at https://doi.org/10.1007/s0024 0-020-01232-4.

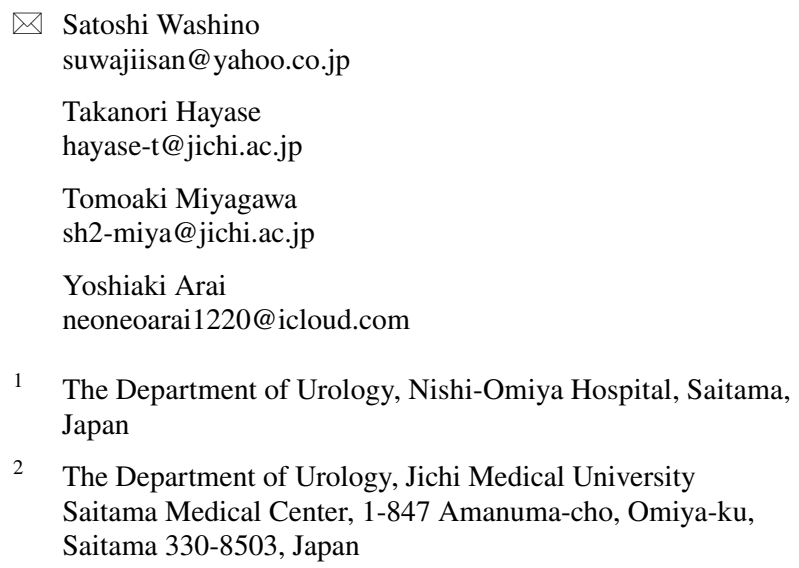

Satoshi Washino

suwajiisan@yahoo.co.jp

Takanori Hayase

hayase-t@jichi.ac.jp

Tomoaki Miyagawa

sh2-miya@jichi.ac.jp

Yoshiaki Arai

neoneoarai1220@icloud.com

1 The Department of Urology, Nishi-Omiya Hospital, Saitama, Japan

2 The Department of Urology, Jichi Medical University Saitama Medical Center, 1-847 Amanuma-cho, Omiya-ku, Saitama 330-8503, Japan

\section{Introduction}

Urinary tract stones are among the most common urological conditions worldwide with estimated prevalence rates of $1-5 \%$ in Asia, 5-9\% in Europe, and 13\% in the USA [1]. Most small stones pass spontaneously or by medical expulsive therapy [2]. The passage rate and spontaneous passage time are affected by the size and position of the stones [3]. Shock wave lithotripsy (SWL) and ureteroscopy lithotripsy (URS) are the main treatments for unpassed ureteral stones [4]. SWL is recommended for proximal and middle ureteral stones, as well as distal stones $<10 \mathrm{~mm}$; URS is recommended for all ureteral stones [5, 6]. SWL can be performed without general or regional anesthesia on an outpatient basis, while URS requires hospitalization and general or regional anesthesia.

In the era of the novel coronavirus disease (COVID-19) pandemic, some patients with ureteral stones may have the virus and medical resources are limited. General anesthesia, 
especially intubation and extubation, is associated with a high risk of COVID-19 transmission [7, 8]; therefore, it is essential to avoid general anesthesia as much as possible. SWL requires fewer medical resources and might pose a lower risk of COVID-19 transmission, compared to URS. Therefore, SWL is becoming increasingly important for the treatment of unpassed ureteral stones.

Early SWL has been reported to yield a higher stone-free rate (SFR), compared to delayed SWL [9]. However, early SWL may constitute overtreatment because some stones pass spontaneously or with medical expulsive therapy. It remains unknown whether urgent SWL is feasible and how long conservative therapies can be continued without compromising SWL outcomes. Here, we studied the association between time to SWL and SFR in patients with ureteral stones undergoing SWL to determine the optimal timing for SWL.

\section{Materials and methods}

\section{Patients}

Patients undergoing SWL for ureteric calculi in Nishi-Omiya Hospital, Japan, between January 1, 2013 and June 30, 2019, were screened for inclusion in this retrospective study. This study was approved by the Internal Review Board of NishiOmiya Hospital (approval no. \#20191206-1). In total, 588 patients were initially included; 51 were excluded for the following reasons: ureteral stent or nephrostomy $(n=24)$; lack of follow-up ( $n=13)$; multiple stones in 1 ureter $(n=9)$; and previous URS and/or SWL for the target stone $(n=5)$ (Fig. 1). Thus, 537 patients were included in the analysis. In total, 491 patients (91.4\%) underwent computed tomography (CT) prior to SWL, but 46 patients did not.

The locations of ureteral stones were classified as proximal (U1), middle (U2), and distal ureter (U3). Patients were grouped according to the time to SWL as urgent

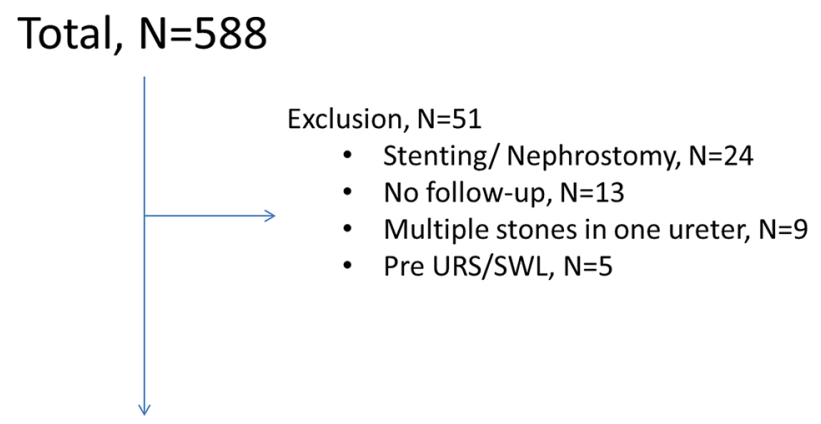

\section{Analyzed, $\mathrm{N}=537$}

Fig. 1 Flow diagram outlining patient selection. URS ureteroscopy lithotripsy, $S W L$ shock wave lithotripsy
(0-3 days), early (4-30 days), late (31-60 days), longdelayed ( $\geq 61$ days), and asymptomatic (unknown). Ureteral wall thickness was measured at the stone site on preoperative non-contrast CT scans, as described previously [10].

\section{SWL methods}

SWL was performed using a Dornier Delta II lithotripter (Dornier MedTech, Munich, Germany). Most patients underwent SWL on an outpatient basis, although older patients or those with one or more severe comorbidities were admitted for SWL. Pain control was achieved using diclofenac sodium (50 mg, suppository) and/or pentazocine hydrochloride (15 mg, intramuscular injection). All SWLs were performed with stepwise power and a shock wave rate of $90-100$ per minute by a single experienced surgeon (Y. A.). Stone fragmentation was determined by $\mathrm{X}$-ray examination. Medical expulsive therapy following SWL was not routinely performed.

\section{Endpoints}

The primary endpoint was SFR, stratified according to time to SWL. The secondary endpoint was SFR following one session of SWL, stratified according to time to SWL. Factors associated with stone-free status were also assessed. Stonefree status was defined as an absence of the target stone on plain X-rays of the kidney, ureter, and bladder. Patients were followed-up every $2-4$ weeks by X-ray until stone-free status was achieved; some patients who did not achieve a stone-free status underwent additional SWL sessions. Comorbidities were graded using the Clavien-Dindo classification [11].

\section{Statistical analysis}

Variables were compared using the $t$ test or Mann-Whitney $U$ test, as well as the $\chi^{2}$ test or Fisher's exact test, as appropriate. Comparisons among three or more groups were performed by one-way ANOVA followed by the Dunnett multiple comparison test or the Kruskal-Wallis test followed by the Dunn multiple comparison test. Binary logistic regression analysis was performed to identify factors independently associated with stone-free status following SWL. Statistical analyses were performed using GraphPad Prism (ver. 7.0; GraphPad, La Jolla, CA, USA) and SPSS Statistics for Windows (ver. 19.0; IBM Corp., Armonk, NY, USA). 
Table 1 Patients' characteristics $(N=537)$

\section{Results}

\section{Patient characteristics}

Patient characteristics are shown in Table 1 . The patients consisted of 402 men (74.9\%) and 135 women (25.1\%), with a mean \pm SD age of $55.5 \pm 13.1$ years. Pain, hematuria, and fever occurred in $77.5 \%, 12.5 \%$, and $3.4 \%$ of patients, respectively; $14.0 \%$ were asymptomatic patients, in whom the finding of ureteral stones was incidental. There had been no prior urolithiasis in $64.6 \%$ of patients, while $35.6 \%$ had at least one previous episode of urolithiasis (Table 1). Stones were present in the right ureter in $46.0 \%$ of patients, whereas they were present in the left ureter in the remaining $54.0 \%$ of patients. Stones were located in U1, U2, and U3 in 57.7\%, $22.4 \%$, and $19.9 \%$ of patients, respectively (Table 2). Hydronephrosis was present in $76.4 \%$ of patients. The mean \pm SD stone size was $7.48 \pm 3.29 \mathrm{~mm}$. U1 stones $(7.77 \pm 3.58 \mathrm{~mm})$ were the largest, followed by $\mathrm{U} 2(7.37 \pm 3.12 \mathrm{~mm})$ and $\mathrm{U} 3$ stones $(6.76 \pm 2.41 \mathrm{~mm})$ (Table 2). Mean \pm SD CT values were $816 \pm 297$ Hounsfield units in the overall population. In total, $28.8 \%, 10.2 \%$, and $31.1 \%$ of all patients were treated with non-steroidal anti-inflammatory drugs, butyl scopolamine, and both drugs in combination to control colic pain before SWL; $29.2 \%$ of the patients were not prescribed any painkiller. For pain control during SWL, $83.7 \%$ and 14.2\% of patients were treated with diclofenac sodium alone or diclofenac sodium plus pentazocine. The mean \pm SD number of shock waves per patient was $2.83 \times 10^{3} \pm 0.40 \times 10^{3}$. The maximum power of lithotripsy was $\leq 2 \mathrm{kV}$ in $12.5 \%$ of patients, $3-4 \mathrm{kV}$ in $57.2 \%$ of patients, $5-6 \mathrm{kV}$ in $30.2 \%$ of patients, and unknown in $0.2 \%$ of patients.
Table 2 Stone characteristics

\begin{tabular}{lll}
\hline & $N$ & $(\%)$ \\
\hline Laterality & & \\
Rt & 247 & $(46.0)$ \\
Lt & 290 & $(54.0)$ \\
Location & & \\
U1 & 310 & $(57.7)$ \\
U2 & 120 & $(22.4)$ \\
U3 & 107 & $(19.9)$ \\
Hydronephrosis & & \\
Yes & 411 & $(76.4)$ \\
No & 50 & $(9.3)$ \\
Unknown & 46 & $(8.6)$ \\
Stone size, mm, mean (SD) & & \\
U1 & 7.77 \\
U2 & 7.37 & $(3.58)$ \\
U3 & 6.76 & $(3.12)$ \\
Total & 7.48 & $(2.41)$ \\
Stone density, CT values (HU), mean & & $(3.29)$ \\
$\quad$ SD) & & \\
U1 & 849 & $(297)$ \\
U2 & 784 & $(278)$ \\
U3 & 755 & \\
Total & 816 & \\
\hline
\end{tabular}

$S D$ standard deviation, $C T$ computed tomography, $H U$ Hounsfield unit

\section{Associations between time to SWL and outcomes}

The mean \pm SD number of SWL sessions was $1.37 \pm 0.92$ in the overall population. Patients in the long-delayed and asymptomatic groups required significantly more SWL sessions, compared to patients in the urgent and early groups $(1.55 \pm 1.10$ and $1.79 \pm 1.08$ vs. $1.24 \pm 0.82, p=0.0002$ and $p<0.0001$, respectively) (Table 3 ).

SFR was $91.6 \%$ in the overall population. SFRs in urgent and early groups were similar (95.2\% and 95.8\%) and decreased with increasing time to SWL. SFRs in long-delayed and asymptomatic groups were significantly lower, compared to urgent and early groups ( $86.3 \%$ and $82.7 \%$ vs. $95.6 \%, p=0.0033$ and $p=0.0008$, respectively), while SFR in the late group (91.3\%) was not significantly different from SFR in urgent and early groups $(p=0.14)$ (Table 4). Long-delayed and asymptomatic groups also had lower SFR following one session of SWL, compared to urgent and early groups (65.3\% and $48.0 \%$ vs. $83.2 \%$; $p=0.0004$ and $p<0.0001$, respectively) (Table 4).

The mean \pm SD time to stone-free status in patients who became stone-free was $24.1 \pm 34.4$ days; this did not significantly differ between urgent and early groups 
Table 3 The number of SWL sessions stratified with time to SWL and stone location

\begin{tabular}{|c|c|c|c|c|c|c|c|c|c|c|c|c|}
\hline \multicolumn{13}{|c|}{ The number of SWL sessions } \\
\hline & \multicolumn{2}{|c|}{$\begin{array}{l}\text { Urgent } \\
\text { (0-3 days) }\end{array}$} & \multicolumn{2}{|c|}{$\begin{array}{l}\text { Early } \\
\text { (4-30 days) }\end{array}$} & \multicolumn{2}{|c|}{$\begin{array}{l}\text { Late ( } 31- \\
60 \text { days) }\end{array}$} & \multicolumn{2}{|c|}{$\begin{array}{l}\text { Long delayed } \\
\text { ( } \geq 61 \text { days) }\end{array}$} & \multicolumn{2}{|c|}{$\begin{array}{l}\text { Asymptomatic } \\
\text { (unknown) }\end{array}$} & \multicolumn{2}{|l|}{ Total } \\
\hline & Mean & (SD) & Mean & $(\mathrm{SD})$ & Mean & $(\mathrm{SD})$ & Mean & (SD) & Mean & (SD) & Mean & (SD) \\
\hline U1 & 1.33 & $(1.08)$ & 1.23 & $(0.87)$ & 1.31 & $(0.87)$ & $1.60 *$ & $(1.37)$ & $1.66^{* *}$ & $(0.94)$ & 1.36 & $(0.99)$ \\
\hline U2 & 1.21 & $(0.58)$ & 1.18 & $(0.58)$ & 1.23 & $(0.44)$ & 1.26 & $(0.55)$ & $1.96 * *$ & $(1.34)$ & 1.37 & $(0.83)$ \\
\hline $\mathbf{U 3}$ & 1.25 & $(0.55)$ & 1.25 & $(0.53)$ & 1.24 & $(0.54)$ & $1.69 *$ & $(1.06)$ & $1.83 * *$ & $(0.94)$ & 1.44 & $(0.80)$ \\
\hline Total & 1.29 & $(0.91)$ & 1.22 & $(0.78)$ & 1.28 & $(0.71)$ & $1.55^{*}$ & $(1.10)$ & $1.79 * *$ & $(1.08)$ & 1.37 & $(0.92)$ \\
\hline
\end{tabular}

$S D$ standard deviation

${ }^{*} p<0.05,{ }^{*} p<0.01$ vs urgent to early SWL (0-30 days)

\begin{tabular}{|c|c|c|c|c|c|c|c|c|c|c|c|c|c|}
\hline & \multicolumn{2}{|c|}{$\begin{array}{l}\text { Urgent } \\
\text { (0-3 days) }\end{array}$} & \multicolumn{2}{|c|}{$\begin{array}{l}\text { Early } \\
\text { (4-30 days) }\end{array}$} & \multicolumn{2}{|c|}{$\begin{array}{l}\text { Late } \\
\text { (31-60 days) }\end{array}$} & \multicolumn{2}{|c|}{$\begin{array}{l}\text { Long delayed } \\
\text { ( } \geq 61 \text { days) }\end{array}$} & \multicolumn{3}{|c|}{$\begin{array}{l}\text { Asymptomatic } \\
\text { (unknown) }\end{array}$} & \multicolumn{2}{|l|}{ Total } \\
\hline & SFR $(\%)$ & $N$ & SFR $(\%)$ & $N$ & SFR $(\%)$ & $N$ & SFR $(\%)$ & $N$ & SFR $(\%)$ & $N$ & & SFR $(\%)$ & $N$ \\
\hline \multicolumn{14}{|c|}{ Stone-free rate $(\%)$} \\
\hline U1 & 96.2 & 52 & 95.9 & 145 & 94.3 & 35 & 92.5 & 40 & $84.2 *$ & 38 & & 93.9 & 310 \\
\hline $\mathrm{U} 2$ & 92.9 & 14 & 97.8 & 45 & 92.3 & 13 & $82.6^{*}$ & 23 & 84.0 & 25 & & 90.8 & 120 \\
\hline U3 & 94.4 & 18 & 91.7 & 24 & 85.7 & 21 & 81.3 & 32 & 75.0 & 12 & & 86.0 & 107 \\
\hline Total & 95.2 & 84 & 95.8 & 214 & 91.3 & 69 & $86.3^{*}$ & 95 & $82.7 *$ & 75 & & 91.6 & 537 \\
\hline \multicolumn{14}{|c|}{ Stone-free rate (\%) following one session of SWL } \\
\hline $\mathrm{U} 1$ & 82.7 & 52 & 84.1 & 145 & 80.0 & 35 & 72.5 & 40 & $52.6^{* *}$ & & 38 & 78.1 & 310 \\
\hline $\mathrm{U} 2$ & 78.6 & 14 & 88.9 & 45 & 69.2 & 13 & 73.9 & 23 & $44.0 * *$ & & 25 & 73.3 & 120 \\
\hline U3 & 77.8 & 18 & 75.0 & 24 & 76.2 & 21 & $50.0^{*}$ & 32 & $41.7 *$ & & 12 & 64.5 & 107 \\
\hline Total & 81.0 & 84 & 84.1 & 214 & 76.8 & 69 & $65.3^{*}$ & 95 & $48.0 * *$ & & 75 & 74.3 & 537 \\
\hline
\end{tabular}

SFR stone-free rate, $N$ number of patients

$* p<0.05,{ }^{* *} p<0.01$ vs urgent to early SWL (0-30 days)
Table 4 Stone-free rate stratified with time to SWL and stone location
$(17.6 \pm 19.4$ vs. $22.0 \pm 33.5$ days, respectively, $p=0.512)$ (Supplementary Table 1). Asymptomatic patients required significantly greater time to achieve stone-free status, compared to patients in the urgent and early groups, while patients in the long-delayed group tended to require more time $(40.8 \pm 60.3$ and $28.1 \pm 35.8$ days in asymptomatic and long-delayed groups vs. $20.0 \pm 28.2$ days in urgent and early groups, $p=0.0012$ and $p=0.080$, respectively).

\section{Associations between other factors and outcomes}

The SFR, and that following one SWL session, were lower for stones $\geq 10 \mathrm{~mm}$ in diameter than for stones $<10 \mathrm{~mm}$ in diameter $(86.4 \%$ vs. $92.9 \%$ and $55.3 \%$ vs. $78.8 \% ; p=0.043$ and $p<0.0001$, respectively); these results were associated with more SWL sessions and a longer time to stonefree status for patients with larger stones $(1.94 \pm 1.61$ vs. $1.24 \pm 0.58$ and $42.0 \pm 55.3$ vs. $20.1 \pm 27.0$ days; $p<0.0001$ and $p<0.0001$, respectively) (Table 5). The SFR, and that following one SWL session, were lower for
U3 compared to U1 stones ( $86.0 \%$ vs. $93.9 \%$ and $64.5 \%$ vs. $78.1 \% ; p=0.014$ and $p=0.0068$, respectively). We found no significant difference in the SFR, number of SWL sessions, or time to stone-free status between patients with and without hydronephrosis $(92.5 \%$ vs. $86.0 \%, 1.34 \pm 0.87$ vs. $1.53 \pm 0.74$, and $23.6 \pm 34.3$ vs. $30.5 \pm 41.7$ days; $p=0.17, p=0.11$, and $p=0.16$ ) but the SFR following one SWL session was higher in patients with than without hydronephrosis $(76.8 \%$ vs. $55.3 \%, p=0.0055)$.

\section{Factors associated with stone-free status in multivariate analysis}

In univariate analysis, factors associated with stonefree status were age (odds ratio [OR], 0.964; 95\% confidence interval [CI], 0.941-0.988; $p=0.004)$, stone size (OR 0.910; 95\% CI 0.843-0.982; $p=0.015$ ), stone location in U3 (OR 0.400; 95\% CI 0.196-0.820; $p=0.012$ ), and time to SWL (long delayed: OR $0.288 ; 95 \%$ CI $0.128-0.645 ; p=0.002$, and asymptomatic: OR 0.218 ; 
Table 5 Association between stone size, stone location, and hydronephrosis with outcomes

Table 6 Univariate and multivariate analyses to analyze factors associated with stonefree status

\begin{tabular}{|c|c|c|c|c|c|c|c|c|c|}
\hline & \multirow[b]{2}{*}{$N$} & \multicolumn{2}{|l|}{ SFR } & \multicolumn{2}{|c|}{$\begin{array}{l}\text { SFR following } \\
\text { one SWL }\end{array}$} & \multicolumn{2}{|c|}{$\begin{array}{l}\text { The number of SWL, } \\
\text { times }\end{array}$} & \multicolumn{2}{|c|}{$\begin{array}{l}\text { Time to stone-free, } \\
\text { days }\end{array}$} \\
\hline & & $\%$ & $p$ value & $\%$ & $p$ value & Mean (SD) & $p$ value & Mean (SD) & $p$ value \\
\hline \multicolumn{10}{|l|}{ Stone size } \\
\hline$<10 \mathrm{~mm}$ & 434 & 92.9 & 0.043 & 78.8 & $<0.0001$ & $1.24(0.58)$ & $<0.0001$ & $20.1(27.0)$ & $<0.0001$ \\
\hline$\geq 10 \mathrm{~mm}$ & 103 & 86.4 & & 55.3 & & $1.94(1.61)$ & & $42.0(53.3)$ & \\
\hline \multicolumn{10}{|c|}{ Stone location } \\
\hline U1 & 310 & 93.9 & ref & 78.1 & ref & $1.36(0.99)$ & ref & $22.5(29.2)$ & ref \\
\hline $\mathrm{U} 2$ & 120 & 90.8 & $0.29^{\#}$ & 73.3 & $0.31^{\#}$ & $1.37(0.84)$ & ns & $29.0(49.8)$ & $\mathrm{ns}$ \\
\hline U3 & 107 & 86.0 & $0.014^{\#}$ & 64.5 & $0.0068^{\#}$ & $1.44(0.80)$ & ns & $23.1(26.1)$ & ns \\
\hline \multicolumn{10}{|c|}{ Hydronephrosis } \\
\hline$(+)$ & 441 & 92.5 & 0.17 & 76.8 & 0.0055 & $1.34(0.87)$ & 0.11 & $23.6 \pm 34.3$ & 0.16 \\
\hline$(-)$ & 50 & 86.0 & & 55.3 & & $1.53(0.74)$ & & $30.5(41.7)$ & \\
\hline
\end{tabular}

SFR stone-free rate, $S W L$ shockwave lithotripsy, ref reference, $n s$ not significant

${ }^{\#} p$ values versus $\mathrm{U} 1$

\begin{tabular}{|c|c|c|c|c|c|c|}
\hline & \multicolumn{3}{|c|}{ Univariate analysis } & \multicolumn{3}{|c|}{ Multivariate analysis } \\
\hline & OR & $95 \% \mathrm{CI}$ & $p$ value & OR & $95 \%$ CI & $p$ value \\
\hline \multicolumn{7}{|l|}{ Age } \\
\hline Years & 0.964 & $0.941-0.988$ & 0.004 & 0.959 & $0.933-0.986$ & 0.003 \\
\hline \multicolumn{7}{|l|}{ Sex } \\
\hline Male & ref & ref & & ref & ref & \\
\hline Female & $(-)$ & $(-)$ & 0.911 & $(-)$ & $(-)$ & 0.480 \\
\hline \multicolumn{7}{|l|}{ Laterality } \\
\hline Rt & ref & ref & & ref & ref & \\
\hline $\mathrm{Lt}$ & $(-)$ & $(-)$ & 0.302 & $(-)$ & $(-)$ & 0.599 \\
\hline \multicolumn{7}{|c|}{ Hydronephrosis } \\
\hline No & ref & ref & & ref & ref & \\
\hline Yes & $(-)$ & $(-)$ & 0.110 & $(-)$ & $(-)$ & 0.779 \\
\hline \multicolumn{7}{|l|}{ Stone size } \\
\hline $\mathrm{mm}$ & 0.910 & $0.843-0.982$ & 0.015 & $(-)$ & $(-)$ & 0.551 \\
\hline \multicolumn{7}{|l|}{ Stone location } \\
\hline U1 & ref & ref & & ref & ref & \\
\hline $\mathrm{U} 2$ & 0.647 & $0.298-1.404$ & 0.270 & $(-)$ & $(-)$ & 0.650 \\
\hline U3 & 0.400 & $0.196-0.820$ & 0.012 & $(-)$ & $(-)$ & 0.049 \\
\hline \multicolumn{7}{|l|}{ Stone density } \\
\hline CT value (HU) & $(-)$ & $(-)$ & 0.647 & $(-)$ & $(-)$ & 0.352 \\
\hline \multicolumn{7}{|c|}{ Distance of skin to stone } \\
\hline $\mathrm{mm}$ & $(-)$ & $(-)$ & 0.630 & $(-)$ & $(-)$ & 0.959 \\
\hline \multicolumn{7}{|l|}{ Time to SWL } \\
\hline$\leq 30$ days & ref & ref & & ref & ref & \\
\hline $31-60$ days & 0.479 & $0.175-1.309$ & 0.151 & 0.417 & $0.146-1.186$ & 0.417 \\
\hline$\geq 61$ days & 0.288 & $0.128-0.645$ & 0.002 & 0.273 & $0.112-0.680$ & 0.004 \\
\hline Asymptomatic & 0.218 & $0.096-0.492$ & $<0.001$ & 0.246 & $0.192-0.593$ & 0.002 \\
\hline
\end{tabular}

$S W L$ shock wave lithotripsy, $O R$ odds ratio, $C I$ confidence interval, ref reference, Hydro hydronephrosis, $C T$ computed tomography, $H U$ Hounsfield unit 
95\% CI 0.096-0.492; $p<0.001)$ (Table 5). In multivariate analysis, age (OR 0.959; 95\% CI 0.933-0.986; $p=0.003$ ) and time to SWL (long-delayed: OR 0.273 ; 95\% CI $0.112-0.680 ; p<0.004$, and asymptomatic: OR 0.218 ; 95\% CI $0.192-0.593 ; p=0.002$ ) were independently associated with stone-free status. Sex, stone laterality, presence of hydronephrosis, stone size, stone location, stone density, and the distance between the skin and the stone showed no significant associations (Table 6).

\section{Association between time from complaint onset and increased ureteral wall thickness on CT}

Ureteral wall thickness was measurable in 454 patients, and was greater in patients with an interval between complaint onset and CT of $\geq 31$ days, and in asymptomatic cases, compared to those within 3 days $(1.98 \pm 0.68,2.41 \pm 0.61$, $2.30 \pm 1.19$, and $3.43 \pm 1.49 \mathrm{~mm}$ vs. $1.78 \pm 0.79 \mathrm{~mm}$ in cases with intervals of $4-30,31-60$, and $\geq 61$ days, and asymptomatic cases vs. those of $0-3$ days; $p=0.26, p=0.0042$ $p=0.0031$, and $p<0.0001$, respectively) (Fig. 2). The SFR did not differ significantly by ureteral wall thickness (92.8\% for a wall thickness $<2.5 \mathrm{~mm}$ vs. $89.6 \%$ for a thickness $\geq 2.5 \mathrm{~mm}, p=0.26$ ) but a thicker wall was associated with a lower SFR following one SWL session, an increase in the number of SWL sessions required, and a longer time to stone-free status $(79.1 \%$ vs. $62.8 \%, 1.31 \pm 0.82$ vs. $1.52 \pm 0.97$, and $23.4 \pm 35.8$ vs. $29.2 \pm 37.0$ days for wall

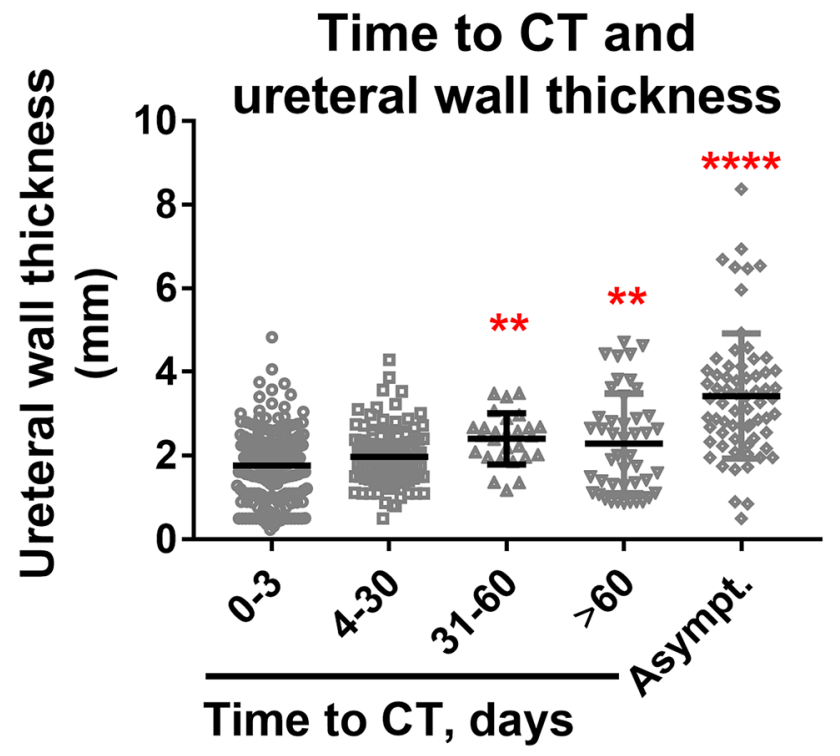

Fig. 2 Association between time from onset to CT and ureteral wall thickness. Ureteral wall thickness increased after 31 days or more from onset, or asymptomatic cases compared to that in 3 days from onset. $* * p<0.01$, ****p $<0.0001$ vs. 0 to 3 days. Asympt. asymptomatic cases thicknesses of $<2.5 \mathrm{~mm}$ vs. $\geq 2.5 \mathrm{~mm} ; p=0.0004, p=0.0006$ and $p=0.0044$, respectively).

\section{Comorbidities}

Twenty-three patients (4.3\%) experienced comorbidities in the overall population; grade 3 or higher comorbidities occurred in only 10 patients (1.9\%), all of which constituted pyelonephritis (Supplemental Table 2). In all 10 patients, pyelonephritis was resolved by antibiotics with or without ureteral stenting.

\section{Discussion}

In the present study, time to SWL was strongly and independently associated with stone-free status following SWL. SFR reached $95 \%$ for patients with $\leq 30$ days to SWL, and then gradually decreased. Patients in the longdelayed ( $>60$ days) and asymptomatic groups required significantly more SWL sessions and showed lower SFR.

The success of SWL reportedly depends on best clinical practices, including clinician experience level and shock wave rate, lithotripter efficacy, stone size, location, and hardness [6]. In the present study, SWL was performed by a single experienced surgeon; therefore, the degree of variation in surgical procedures was low. We explored factors related to stone-free status, following SWL. The results indicated that time to SWL and age were independently negatively associated with stone-free status, while stone size, stone density (CT value), the distance from the skin to the stone, and stone location showed no associations in multivariate analysis (Table 6). Ichiyanagi reported that older age tended to be associated with delayed stone clearance following SWL [12]. There have been no studies regarding the association between time to SWL and SFR; the outcomes of SWL in patients with asymptomatic ureteral stones have not yet been determined, although patients with urgent SWL have a higher SFR, compared to patients with delayed SWL [9]. The present study demonstrated that longer time to SWL and asymptomatic ureteral stones were negatively associated with SFR. Therefore, time to SWL and age should be considered with respect to SWL for the treatment of ureteral stones.

The aim of emergent treatment in patients with acute renal colic is relief of symptoms, unless there are indications for immediate intervention (e.g., renal function impairment and signs of urinary tract infection or sepsis) [13]. Kumar et al. [14] reported that the time needed for stone clearance, the retreatment rate, and the requirement of auxiliary procedures were significantly higher when treatment was performed beyond $48 \mathrm{~h}$ after onset of pain. In a meta-analysis, emergent SWL was found to 
be more effective than delayed SWL in terms of SFR; it also required fewer ancillary procedures [9]. However, most small ureteral stones passed spontaneously or with medical expulsive therapy. An estimated $95 \%$ of stones up to $4 \mathrm{~mm}$ pass in $\leq 40$ days with conservative treatment; medical expulsive therapy seems to be efficacious for treatment of patients with ureteral stones, especially distal stones $>5 \mathrm{~mm}$ [2]. Choi et al. [15] reported significantly better treatment outcomes in the emergent group only for patients with stone size $<10 \mathrm{~mm}$ and proximal stones. In a randomized controlled trial, Kumar et al. [14] found no significant difference in SFR between patients with delayed SWL and those with early SWL, although the numbers of auxiliary procedures and SWL sessions were significantly lower in patients with early SWL. The usefulness of emergent SWL remains controversial with respect to SFR. In the present study, SFR remained up to $95 \%$ for $\leq 30$ days and we found no difference in the time to stone-free status between patients in the urgent (0-3 days) and early groups (4-30 days) (Table 4 and Supplementary Table 1). These results indicate that it is feasible to continue conservative therapy or medical expulsive therapy for up to 30 days in patients without severe pain and/or infection.

Patients in the long-delayed (time to SWL $>60$ days) and asymptomatic groups had significantly lower SFR and required more SWL sessions, compared to patients in the urgent and early groups (Tables 3 and 4). Especially in patients with distal stones (U3 stones), SFR could decline to $~ 80 \%$; SFR following one session of SWL was only 40-50\% (Table 4). Ureteral stones cause ureteral edema that develops gradually after $24-48 \mathrm{~h}$, progressing over time; these stones ultimately impact the ureteral wall, resulting in impaired stone clearance [16]. We found that ureteral wall thickness, as revealed by CT, increased significantly more than 1 month after onset (Fig. 2), which compromised stone clearance following SWL. It is feasible to avoid continued use of conservative therapy or medical expulsive therapy for more than 2 months if SWL is considered as an alternative approach. If time to surgical treatment is expected to be $>2$ months, URS may be preferable; notably, SFR is less affected by time to surgical procedure in patients undergoing URS [9].

This study had some limitations. In particular, the retrospective nature of this study increased the patient selection bias and the number of patients in some groups was small, especially those in the late and asymptomatic groups. Furthermore, this study did not assess the retreatment rate.

\section{Conclusion}

Time to lithotripsy is strongly associated with SFR following SWL. SWL in an urgent care setting does not improve SFR if it is performed within 1 month, while time to $\mathrm{SWL}>2$ months reduces the likelihood of stone-free status.

Author contributions SW and YA contributed to the study conception and design. Material preparation, data collection and analysis were performed by SW and TH. The first draft of the manuscript was written by SW, TM and YA and all the authors commented on previous versions of the manuscript. SW edited this manuscript. All the authors read and approved the final manuscript.

Funding Non-applicable.

\section{Compliance with ethical standards}

Conflict of interest The authors declare that they have no conflict of interest.

Human and animal rights This article does not contain any studies with human participants or animals performed by any of the authors.

\section{References}

1. Ramello A, Vitale C, Marangella M (2000) Epidemiology of nephrolithiasis. J Nephrol 13(Suppl 3):S45-50

2. Segura JW, Preminger GM, Assimos DG et al (1997) Ureteral Stones Clinical Guidelines Panel summary report on the management of ureteral calculi. J Urol 158:1915-1921

3. Miller OF, Kane CJ (1999) Time to stone passage for observed ureteral calculi: a guide to patient education. J Urol 162:688-691

4. Pearle MS, Calhoun EA, Curhan GC (2005) Urologic disease in America project: urolithiasis. J Urol 173:848-857

5. Assimos D, Krambeck A, Miller NL et al. (2016) Surgical management of stones: AUA/Endourology society guideline. https:// www.auanet.org/guidelines/kidney-stones-surgical-management -guideline. Accessed 2 Nov 2020

6. Türk C, Neisius A, Petrik C et al (2020) EAU guideline for urolithiasis. https://uroweb.org/guideline/urolithiasis/. Accessed 2 Nov 2020

7. Dondorp AM, Hayat M, Aryal D et al (2020) Respiratory support in novel coronavirus disease (COVID-19) patients, with a focus on resource-limited settings. Am J Trop Med Hyg 102:1191-1197

8. Asenjo JF (2020) Safer intubation and extubation of patients with COVID-19. Can J Anesth 67:1276-1278

9. Arcaniolo D, De Sio M, Rasseiler J et al (2017) Emergent versus delayed lithotripsy for obstructing ureteral stones: a cumulative analysis of comparative studies. Urolithiasis 45:563-572

10. Sarica K, Kafkasli A, Yazici Ö et al (2015) Ureteral wall thickness at the impacted ureteral stone site: a critical predictor for success rate after SWL. Urolithiasis 43:83-88

11. Dindo D, Demartines N, Clavien PA (2004) Classification of surgical complications: a new proposal with evaluation in a cohort of 6336 patients and results of a survey. Ann Surg 240:205-213

12. Ichiyanagi O, Nagaoka A, Izumi $T$ et al (2015) Age-related delay in urinary stone clearance in elderly patients with solitary 
proximal ureteral calculi treated by extracorporeal shock wave lithotripsy. Urolithiasis 43:419-426

13. Türk C, Petř́ík A, Sarica K et al (2016) EAU guidelines on diagnosis and conservative management of urolithiasis. Eur Urol 69:468-474

14. Kumar A, Mohanty NK, Jain M et al (2010) A prospective randomized comparison between early $(<48 \mathrm{~h}$ of onset of colicky pain) versus delayed shockwave lithotripsy for symptomatic upper ureteral calculi: a single center experience. J Endourol 24:2059-2066

15. Choi HJ, Jung JH, Bae J et al (2012) Usefulness of early extracorporeal shock wave lithotripsy in colic patients with ureteral stones. Korean J Urol 53:853-859
16. Deliveliotis C, Chrisofos M, Albanis S et al (2003) Management and follow-up of impacted ureteral stones. Urol Int 70:269-272

Publisher's Note Springer Nature remains neutral with regard to jurisdictional claims in published maps and institutional affiliations. 